\title{
The Bernoullis and the Origin of Probability Theory: Looking back after 300 years
}

\section{Wolfgang Polasek}

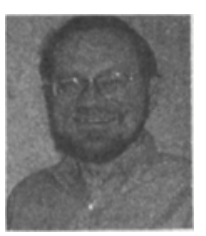

Wolfgang Polasek graduated from the University of Vienna in 1976 with a degree in statistics. He held visiting positions at the University of Southern California, the Institute of Advanced Studies, Vienna and the Institute of Mathematical Statistics, Tokyo before he became a professor of statistics and econometrics at the University

Basel in 1987. His major research interests are multivariate time series models, Bayesian and financial econometrics and computer-intensive statistical methods.
This paper describes the contribution of the four famous Bernoullis (Jacob, Johann, Daniel and Nicolaus) to the development of the theory of probability between 1670 and 1760. Their contributions are evaluated from a modern day position of probability theory and statistics. We argue that abstract formulation of problems and mathematical solution concepts for abstract problems and seemingly special questions have a long term impact on the diversification of science. In the appendix, reference is also given to the ongoing work of the Bernoulli Edition in Basel.

\section{Introduction}

In the year 2000, we celebrate the 300th birthday of Daniel Bernoulli, the youngest of the three great Bernoullis who lived in the 17th and 18th centuries in Basel. Leibniz's introduction of the differential calculus in 1684 led to a revolution in solving applied mathematical problems. Jacob and Johann were not only the first ones who understood the power of the new approach, they also contributed to the development of the new theory. Despite the fact that Jacob created a completely new approach to probability theory, the power of mathematics did not trigger so much progress in statistics, it helped first to develop mathematics, physics and natural sciences. The development of probability theory in the Ars Conjectandi seems to have been - economically speaking - a fringe benefit or an external effect of the new mathematical thinking. It took many more generations of scientists to digest this 'exact approach to uncertainty' than to apply differential calculus to complicated but well posed problems in deterministic models of sciences. Jacob Bernoulli deserves to be regarded as the first researcher who saw that it is possible to make precise statements about 
uncertainties of events which are basically random. While combinatorics was known to be used to calculate probabilities for a well defined experiment, it was less clear that probabilities could also be measured from certain random sequences in an arbitrarily precise way.

1) Through the work of Huygens, it became necessary to make the terms 'probability' and 'expectation' more precise, to describe their meanings and explain them mathematically. Jacob Bernoulli's wish for 'moral certainty' led to the law of large numbers and to the definition of stochastic convergence. The 'moral value' of a game with infinite outcomes led Daniel Bernoulli to the new concept of subjectively perceived value or expected utility (in today's language).

2) Combinatorics is a way to determine probabilities 'a priori' before any data are seen. The law of large numbers allows us to measure probabilities 'a posteriori' - after the data have been collected in repeated random experiments. Ultimately the law of large numbers led to the frequentist definition of probability in the 20th century and to the frequentist interpretation of statistical inference which dominates statistical practice 300 years after the Bernoullis.

3) New mathematical methods are not only important for the development of abstract mathematics, they cross-fertilize all disciplines of science, sometimes with a long time delay. Useful concepts in one family of problems trigger similar approaches in related problems of other fields.

4) The long term yield of a scientific approach might not be realized completely by contemporary researchers. The more abstract a new solution is, the longer it takes to be understood and applied or to create complete new roads of developments. Such new research results do not only accumulate knowledge of a society but eventally contribute to more public utility in future. Some results might even be of no immediate use or could be rather unimportant for the branch of science where they were found. Note that early probability contributions preceded the

The long term yield of a scientific approach might not be realized completely by contemporary researchers. 
Structuring knowledge in the right framework and posing solvable classes of problems, can make originally complicated abstract results more understandable and practically useful. work of what we call 'statistics' today. This term was invented around 1780, i.e., about 100 years after Ars Conjectandi was conceived by Jacob Bernoulli. Also, the term 'distribution of a random variable' was not known. Applying the Chebyshev inequality on distributions, the law of large numbers can be proved very easily. This shows that general progress in science, i.e., structuring knowledge in the right framework and posing solvable classes of problems, can make originally complicated abstract results more understandable and practically useful. In the following sections, we briefly describe the contributions of the Bernoulli scientists to the development of probability theory in the 17th and 18th centuries and their relationships to other mathematicians at their time.

\section{Jacob Bernoulli}

Jacob Bernoulli was born on December 27, 1654 in Basel to a druggist and Margarethe Schnauer, the daughter of a Basler banker. (Note that all the dates before December 31, 1700 are Julian dates while the year 1701 started with the 12th of January as new Gregorian calendar in Basel, Bern and Zurich.) His grandfather moved from Frankfurt/Main to Basel (after the Huguenot family had left Antwerp, Netherlands at the end of the 16th century), where he became a citizen in 1622 . Jacob (translated in English as James, in French as Jacques) studied philosophy and theology at the University of Basel, where he obtained the masters degree in philosophy in 1671 and the 'licenciat' in theology in 1676. After his studies he was a private teacher in Geneva and he spent the period 1677-1680 in France where he published a paper on solar watches (tabula gnomonicae universalis). Since his student days he had been interested in astronomy and when the Kirch comet appeared in 1680 he followed up an idea that comets were satellites of a transSaturnian planet which led to his first scientific paper. He spent 1681-1682 in Netherlands and England meeting scientists and writing his scientific diary (Meditationes). After returning to Basel in 1683 he gave private lectures on experimental physics and he published his first work on a probability problem in 
1685. He found his brother Johann Bernoulli to be a good student and a discussion partner, as Johann had studied arts and medicine at his father's orders. In 1685 Johann finished his master of arts degree.

The brothers studied the new infinitesimal calculus of $G W$ Leibniz (1646-1716), which had just been published in 1684. In the words of G Shaffer (1982, p. 215): "They were the first to master Leibniz's method, and it was through the brilliant papers that they and Leibniz published from 1690 onwards that calculus became known to the learned world". In 1687 Jacob Bernoulli became a professor at the University of Basel and, despite his discontent with university affairs, he worked on the theory of series, the law of large numbers and complete induction. According to the comments of van der Waerden [5], the law of large numbers, which was simply called the 'main theorem', was proved almost certainly in the years $1687-1689$, just after Jacob Bernoulli was appointed professor at the University of Basel. The work on his 'main theorem' started around 1684, which can be documented by written letters. The name law of large numbers' was given much later by Poisson in 1837. The dating of the discovery of the theorem can be traced by Jacob's own testimony just before his death: 'A problem in which I've engaged myself for 20 years'.

\subsection{The story of Ars Conjectandi}

The story of Ars Conjectandi really starts with Christiaan Huygens (1629-1695) who wrote the book Van Rekeningh in Spelen van Geluck (calculation in hazard games) in 1657. This was translated into Latin by Frans van Schooten (1615 -1660), a professor at the University of Leiden and a teacher of Huygens, with the title: De Ratiociniis in Ludo Alea. The paper, originally written in Dutch, appeared in the appendix of Exercitationum mathematicarum libri quinque. At the end of this paper Huygens posed five problems. Before Jacob started to write his book, he solved all the five problems. This led van der Waerden (1975) to the following first sentence of Vol. 3 in the collected works of Jacob
Jacob and Johann Bernoulli were the first to master Leibniz's method, and it was through the brilliant papers that they and Leibniz published from 1690 onwards that calculus became known to the learned world. 
The probability theory was created by Christiaan Huygens and Jacob Bernoulli out of nothing.
Jacob Bernoulli's scientific aim was not to develop a new theory but to solve certain practical problems and to show their applicability.
Bernoulli: 'The probability theory was created by Christiaan Huygens and Jacob Bernoulli out of nothing'. Others had touched on problems of games of chance but no one had created a logically founded theory. It seems that Jacob Bernoulli started with his book project around 1685 , but it remained unfinished. The book Ars Conjectandi is written in Latin and the title means, literally translated, 'art of conjecturing' and has been translated in English and German to 'probability theory'. It is remarkable that Jacob emphasized the fact that solving probability problems is a matter of art. Generally, it seems that Jacob Bernoulli's scientific aim was not to develop a new theory but to solve certain practical problems and to show their applicability (in the same way as the newly invented infinitesimal calculus of Leibniz could solve completely new classes of problems). In this context many researchers and historians point to the fact of how abruptly the Ars Conjectandi ends: Stigler (1988) also notes in his book on the history of statistics that Jacob Bernoulli was concerned with the 'moral certainty' of - what we call now - the sample size of an urn problem. Since this example had a major influence on the understanding of statistics we want to describe it in our present statistical language.

Suppose we know that an urn consists of 3000 white and 2000 black pebbles and we want to recover this proportion empirically up to a small error term, say $\pm 1 / 50= \pm 0.02$, by drawing pebbles randomly and repeatedly from the urn. Consider now the event that the empirical frequency deviates from the true proportion by 0.02 . Note that this can be viewed as a probability statement in the meta-language: The target is not the value of the proportion, but the deviation of the empirical from the true proportion. Now we look at a hypothetical event that the empirical proportion lies in the neighborhood of the true proportion or it does not. The ratio of these two probabilities is today called 'odds of the event' (over the contrary event). A probabilistic notion of precision of measurement was now given by Jacob Bernoulli: Look at repeated trials (of the same kind) and find out if in more and more trials, the empirical frequency lies 
within the prespecified target range than outside. Clearly, big odds are connected only with a long repetition of the experiment. How many draws are necessary in order to make the odds of this event large, say 1000? Or, in the words of Jacob Bernoulli: 'Can you do this so often that it becomes 10 times, 100 times, 1000 times, etc. more probable (that is, morally certain) that the number of whites and blacks drawn are in the same ratio as in the urn (3:2) rather than any other different ratio?' (see appendix A). For an odds ratio of 1000 Jacob calculated the number 25,500. This number seems to have been too big for him and he had trouble digesting it. Maybe this was the reason he stopped working on his book. Stigler argues that the number 25,500 was too big to be acceptable for J Bernoulli at that time to make meaningful (morally acceptable) comparisons: for example, the population of Basel at that time was less than 25,000 and less than 3000 stars were known at his time. The last paragraph of the book is a rather philosophical reflection of his results. He cites Plato's theory of the general circulation phenomenon that after innumerable centuries all things will revert to the predicted state (reversura statum praedixit): The probability finally goes into perfect certainty (probabilitate ultimo in perfectam certitudinem abeunte).

Was this the origin of the main theorem of J Bernoulli being named the law of large numbers? Note that Jacob used the word 'probability' in the last paragraph after he started with words like 'verisimilis' or 'sors'. The reason for this seems to trace back to van Schooten who had difficulties translating Dutch expressions of randomness into Latin. Van der Waerden ([5] p.11) remarks that van Schooten translated the word 'randomness' (Dutch: geval) into 'sors' and not into 'fortuna' (the word Huygens wanted). He did not translate the word 'chance' (Dutch: kansse); he avoided it by circumscribing it depending on the situation.

When did Jacob start writing his book? Johann Bernoulli wrote in a letter to Leibniz on February 16, 1697: My brother has been working for many years on a book which he calls Ars Conjectandi. From the Meditationes it is known that all preliminary work was 
In games which are ruled by chance the events are uncertain; but it is possible to determine how much closer someone is to win than to lose. finished in 1690 . Why did Jacob leave the book unfinished? Many people thought that Jacob was not convinced by his examples. Did he delay his work because he looked for convincing examples? In the last years before his death he worked again on his book. On October 3, 1703 he wrote to Leibniz: 'I have completed the larger part of my book, but the most important part is missing, where I show how the basics of Ars Conjectandi can be applied to civil, moral and economic matters'.

The publication process after his death was delayed because the family was suspicious about Johann and the fact that in the last years the brothers were involved in fights over mathematical originality and the relationship turned bitter. Jacob's only son, a painter, started to bring together all the related material with the help of Varignon, the faithful disciple of the Bernoullis. Nicolaus was the editor of the whole project.

\subsection{On the Significance of Facob Bernoulli's Work}

A modern appraisal of Jacob Bernoulli's Ars Conjectandi is given in Shaffer (1996). In the following I want to highlight some points which have not found attention in the literature so far. It is interesting that Jacob started his book with the definition and justification of odds: 'In games which are ruled by chance the events are uncertain; but it is possible to determine how much closer someone is to win than to lose'. It is also worth mentioning that he does not use a single word for probability but he uses different expressions depending on the situation. 'Closer' (propior) could be translated as 'likely'. But in the next sentence he uses the word 'verisimilis' (probable) which translates directly into the German word 'wahrscheinlich' (literally: truth similar).

What is the important contribution of Ars Conjectandi? The first part of the book demonstrates how to solve the five problems of Huygens. The second part develops the theory of combinatorics and the third part shows how to use combinatorics for problems of probability. The fourth part is the shortest but is certainly the most influential, since it contains the 'main theorem'. To appreciate the scope of the theorem one has to remember that prob- 
ability theory was no formal theory at all at that time. People had the notion of determining chances by mathematical tools, like combinatorics, and called them 'a priori probabilities' since they are determined without looking at empirical facts. The law of large numbers was seen to be an instrument for determining probabilities 'a posteriori', i.e., from empirical observations. If it is not possible to determine probabilities a priori then one can find them by observing an experiment long enough and Jacob Bernoulli's theorem (the law of large numbers) makes sure that you can measure probabilities with increasing precision, even if it takes a very, very ('eternally') long time. The history of statistics has shown that much time had to elapse before it was possible to implement this idea in practice, as for example we use it today. The notion of precise reasoning is an ideal, difficult to rationalize and very complicated to implement in everyday life. Jacob Bernoulli published about 60 papers but no books. Because of his scientific success he became a member of the Paris Academy of Science in 1699. The book Ars Conjectandi appeared in 1713, eight years after his death on August 16,1705, with the help of his cousin Nicolaus and his brother Johann, who succeeded him in 1705 as a professor at the University of Basel. The epitaph of Jacob Bernoulli can be found in the cloister of the 'Muenster' in Basel and in the bottom part of the frame one can see the logarithmic spiral with the words: Eadem mutata resurgo (I resurrect changed in the same way).

This is an homage to the eternal cycle of life which is also today the logo of the Bernoulli society and appears on the front of the Bernoulli journal. Jacob Hermann (1678-1733), a scholar of the Bernoullis', published a necrology on the achievements of Jacob Bernoulli in Acta Eruditorum in 1706. Leibniz - honoring Jacob's contributions to the differential calculus - added to this: 'The new calculus merits being named after the two Bernoullis with the same right as being named after himself.

\subsection{The Law of Large Numbers Today}

In modern language the law of large numbers says that the
The new calculus merits being named after the two Bernoullis with the same right as being named after himself. 
Though the law of large numbers was invented (or detected) about 300 years ago, even today we do not understand the implications of the theorem any better than the mathematicians who tried to understand the main theorem of Ars Conjectandi. relative frequency of successes in independent repetitions of Bernoulli experiments (i.e., a random variable with only two states of outcomes called success and failure) converges in probability to the unknown probability of success if the number of trials increases indefinitely. This is a new concept of weak convergence in probability, since the mathematical concept of convergence applies only to deterministic sequences. Though the law of large numbers was invented (or detected) about 300 years ago, even today we do not understand the implications of the theorem any better than the mathematicians who tried to understand the main theorem of Ars Conjectandi. There are at least three reasons why the meaning and interpretation of the law of large numbers is often misunderstood, even today: 1) it concerns random experiments; 2 ) it is about stochastic convergence, and 3) it is a statistical meta-statement, the probability (odds) about a probability, the parameter of the Bernoulli distribution. The last mentioned is the reason why we describe the consistency of estimators today by analogy with the law of large numbers: A statistical estimator is consistent if it approaches, in the limit, the unknown (true) parameter of the population.

Here are some common caveats: By the law of large numbers you can't improve the uncertainty of the basic random experiment. You only know that you are measuring the basic probability with more and more precision. Even this creates many confusions. The law only states the convergence of the empirical proportion if the observations are generated from a constant true process. But in absolute terms the deviations can become larger and larger and the improvement in precision does not always hold for absolute values. Many lotteries publish tables which show the frequency of the numbers drawn. The implicit appeal is that numbers which are drawn less might have a better chance to be drawn next or numbers which have occurred too often are not likely to show up in the near future. It is very difficult to explain to laymen that this is not a consequence of the law of large numbers. The basic random drawing mechanism which is independent of the past stays the same. The 
chance to draw the number which occurred least (or most) is exactly the same as it was for the first time or any other time in the past. The law of large numbers just states that the measurement of the underlying parameters of the random experiment can be made more precise - in relative terms. It could be that Jacob Bernoulli was the first one to see this new road of science which led to a probabilistic revolution in empirical research work in the last century. Loosely speaking, the law of large numbers has a learning interpretation from a philosophy of science point of view: Working with observations which are exposed to uncertainty and measurement errors will not create knowledge accumulation with absolute certainty. In the words of Jacob Bernoulli, empirical knowledge has to be judged with 'moral certainty'. Like the definition of convergence of series, moral certainty is a concept which implies a thought experiment that probabilities could possibly be measured with increasing precision. But the price to pay is that the number of experiments which have to be repeated under the same conditions could become unrealistically large to be useful as a practical concept. This shows that the frequentistic notion of probability has its limits: How should we measure the probability of an event where a repeated empirical measurement is not possible? It was about 100 years later that Thomas Bayes $(1702-1761)$ and Simon Laplace (1749-1827) found other notions of probability which gave a totally different interpretation for 'a priori' and 'a posteriori' probabilities.

\section{Johann Bernoulli}

Johann Bernoulli (6.8.1667 - 1.1.1748) was the tenth child and was found to be unsuitable for a business career by his father. Therefore he was allowed to study medicine (until 1690) at the University of Basel and later he started studying mathematics with his 12-year-older brother Jacob who had obtained the mathematics chair in 1687. During 1691-92 he visited Geneva and Paris and gave lessons to the Marquis de l'Hospital (16611704). The correspondence that followed on the new 'Leibniz calculus' led to the first textbook in differential calculus by de
The law of large numbers just states that the measurement of the underlying parameters of the random experiment can be made more precise - in relative terms. 


\begin{abstract}
After Newton's
death in 1727 , Johann Bernoulli was unchallenged

as the leading mathematical preceptor to all Europe.
\end{abstract}

l'Hospital (Analyse des infiniment petits). Later (1721) it was found that there is a very closely related manuscript by Johann called Lectiones de calculo differentialium in the University Library at Basel.

In 1692 Johann met Pierre Varignon who later became his disciple and a close friend. In 1695 Johann was offered (by the intervention of Huygens) a chair of mathematics in Groningen. After having worked on integration (viewed as inverse operation of differentiation) he published his solution of the problem of the brachistochrone in 1696 (i.e., how to find the 'curve of the steepest descent'). One solution was given in 1690 by his brother Jacob (Leibniz, Newton and Huygens also gave solutions to the 'chain-line' problem) which ultimately led to the conflict between the two brothers. Johann was the first to define explicitly the notion of a function in 1718. His best known student was Leonhard Euler (1707-1783).

Since Johain's area of interest was not in the field of probability, we do not fully describe his work in this paper. We only want to mention his outstanding contributions to modern mathematics, since it clarifies his relation to the other Bernoullis who worked on topics of probability theory. A final citation from Gillispie (1970) sheds some light on Johann's personality:

'After Newton's death in 1727, Johann Bernoulli was unchallenged as the leading mathematical preceptor to all Europe. Since his return to Basel in 1705, he had devoted himself - in the field of applied mathematics - to theoretical and applied mechanics. ... Johann Bernoulli's quarrelsomeness was matched by his passion for communicating. His scientific correspondence comprised about 2500 letters, exchanged with some 110 scholars.'

\section{Daniel Bernoulli}

Daniel Bernoulli (8.2.1700 - 17.3.1782) was the most gifted son of Johann and was born in Groningen when his father was a professor there. He studied medicine in Basel, Heidelberg and 
Strasbourg and wrote a dissertation de respiratione on the mechanics of breathing in 1721 . He loved to study mathematical problems and he published his exercitationes mathematicae in Venice in 1724, a result of his studies in Italy, which consists of four rather independent topics. One topic was the integration of a differential equation which was later named after Riccati (1676 -1754). Nevertheless the work attracted so much attention that in 1725 he was offered a position in St. Petersburg (for mechanics) at the newly founded Academy of Science. The story goes that it was not clear which Bernoulli got the offer from the Academy. It could also have been Nicolaus II (born in 1695) who could speak 4 languages at the age of 8 and who was a professor of law in Bern. So, their father Johann I asked for clarifications and as a result both the Bernoulli brothers got offers from the Academy. Daniel travelled with his elder brother Nicolaus II to work in St.Petersburg in 1725. Unfortunately, Nicolaus II died after one year at the age of 31 from.fever and this led Daniel Bernoulli to look for other appointments. In 1733 he returned to Basel after eight very fruitful years at the Imperial Academy in St.Petersburg. At the University of Basel, he first got the chair for anatomy and botany and the chair for physics in 1750. His main work is the book Hydrodynamica which was completed by 1734 but not published until 1738. In this book the basic equation, Bernoulli equation of fluid dynamics is given. His father Johann I Bernoulli wrote about the same time the book Hydraulica which appeared in 1742 and he predated it to 1732 - just to ensure priority for himself. To Daniel's big surprise he found in this book several results related to his own work. Daniel got the impression that his father wanted him to feel that he had known these results much earlier. Since Daniel saw himself in the position of a plagiarist, he wrote in a letter to L Euler: 'I feel robbed of my total Hydrodynamica for which I owe not an iota to my father and I have lost in a single moment the fruits of a 10-year long work'. After Daniel left St.Petersburg, he began an intensive and fruitful scientific collaboration with L Euler another citizen of Basel) between 1726 and 1767. The other two main scientific letter exchanges were with Christian
I feel robbed of my total Hydrodynamica for which I owe not an iota to my father and I have lost in a single moment the fruits of a 10-year long work.' 
What is the fair price of a game with infinite mathematical expectation?
Are the angles of the planes of the planets around the sun randomly distributed?
Goldbach (1690-1764) and his nephew Johann III (1744-1807). Daniel Bernoulli had three rather different scientific working fields: medicine, mathematics including probability theory, and physics, especially mechanics. Here we concentrate on his contributions to probability theory and statistics. His most important work in this area was the book 'Specimen Theoriae Novae De Mensura Sortis' (literally: Exposition of a new theory for the measurement of chance; but often just called Probability Theory) published in 1738 which was started during his stay in St. Petersburg. An English translation of 'De Mensura Sortis' appeared in Econometrica (1954), Vol.22, 23-36. Here he started the theory of utility called 'emolumentum' in Latin (see Vol. 2 of the collected work of D Bernoulli) but also called 'moral value', 'moral expectation' (or in older versions 'hope'). The idea for this paper was from his cousin Nicolaus: What is the fair price of a game with infinite mathematical expectation? (Recall the 'St.Petersburg Paradox' which appeared in Resonance, Vol. 1, No. 9, 1996 and Vol. 2, No. 1, 1997.) In 1760 he treated a medical problem (today: epidemiology) in a similar way: What is the rate of mortality of smallpox across age groups? He touched also the realm of demography by determining the average length of a marriage for every age. He also wrote a paper on the question: Are the angles of the planes of the planets around the sun randomly distributed? The solution he offered was obtained by what we call today a geometrical probability approach. The maximum observed difference was an angle of 6 degrees and 54 '. What is the probability that the other planes fall in this interval 'randomly'? Daniel Bernoulli also won 10 prizes (some of them shared) for the competition of the Paris Academy which shows the popularity of his research work.

\section{Nicolaus Bernoulli I}

Nicolaus Bernoulli I (October 21, 1687 - November 29, 1759) was the son of Nicolaus Bernoulli and studied mathematics with his two uncles, Jacob and Johann. He started his dissertation $D e$ usu artis conjectandi in jure (the use of probability theory in law) in 1704 with Jacob Bernoulli. He finished his 'licenciat' at the 
law faculty in 1709 and became a doctor of law in 1717. He taught mathematics at the University of Padova during 17161719 and became a professor of logic at the University of Basel in 1722 and a professor of law in 1731. In the priority dispute of the infinitesimal calculus he took (with his uncle Johann) the side of Leibniz by claiming that Newton misunderstood higher order derivatives. (Johann Bernoulli used higher order derivatives for his expansions.) He finished editing Ars Conjectandi in 1713 after Jacob's son, a painter, gave up on this project which should have been originally printed in Paris. In 1744 he made notes on the letter exchanges of Jacob with Montmort, on topics which we call today optimal solutions in game theory. Honouring his contributions, Nicolaus' dissertation (in Latin) is also printed in volume 3 of the Bernoulli Edition next to the (Latin) Ars Conjectandi of Jacob.

Note: Nicolaus Bernoulli is counted as 'one of the smaller Bernoullis' compared to the 'big Bernoullis': Jacob, Johann and Daniel. We only mention him here because of his direct involvement with his uncle Jacob's book Ars Conjectandi. More details on the work of the other Bernoullis can be obtained from the Bernoulli Edition in Basel, a research project connected with the University Library in Basel (see Appendix).

Appendix. A. Jacob Bernoulli's formulation of the Law of Large Numbers

Consider an urn with $r$ white and $s$ black pebbles. Jacob's formulation was to find in a repeated experiment the number $N$ for which it is 'morally certain' that the ratio $r /(r+s)$ is recovered up to a small error, say $\pm \varepsilon= \pm 1 /(r+s)$. In his words (1713, in a modified translation of S Stigler, 1986): 'To illustrate this by an example, I suppose that without your knowledge there are concealed in an urn 3000 white and 2000 black pebbles and, to determine the number of these pebbles you take out one pebble after another (each time replacing the pebble you have drawn in order not to decrease the number of pebbles in the urn), and that you observe how often a white and how often a 
black pebble is drawn. The question is, can you do this so often that it becomes 10 times, 100 times, 1000 times, etc. more probable (that is morally certain) that the number of white and black pebbles chosen are in the same 3:2 ratio as the pebbles in the urn, rather than any other different ratio?'

Let us assume that a random variable $X$ counts the number of white pebbles drawn after $N$ draws and that $N$ is a multiple of $r$ $+s$, where $n$ is the multiplication factor, i.e., $N=n(r+s)$. Furthermore, the unknown ratio of pebbles in the urn is $p$ and the error margin is then $\varepsilon=n / N$. Now we consider the odds of an event, the ratio of the probability of the event against the contrary event:

$$
\text { Odds }(A)=\operatorname{pr}(A) /(1-\operatorname{pr}(A)) .
$$

If $\operatorname{pr}(A)=0.9$ then Odds $=9$ (or 9:1), i.e., event $A$ is 9 times more likely than the opposite event. The law of large numbers formulated in odds is

$$
\text { Odds }(|X / N-p|<\varepsilon)>\text { c. }
$$

Now, Jacob calculates $N$ for different urn compositions and increasing the odds $c$ by the factor 10 and he gets the following results:

$\begin{array}{lllll}r & s & \varepsilon & c & N \\ 30 & 20 & 1 / 50 & 1000 & 25,550 \\ 300 & 200 & 1 / 500 & 10,000 & 31,258 \\ 3000 & 2000 & 1 / 5000 & 100,000 & 36,966\end{array}$

At this point Jacob stops his book.

\section{Appendix B. The Bernoulli Edition, Basel}

(http://www.zuv.unibas.ch/bernoulli)

The following description is taken from the latest leaflet of the Bernoulli Edition, Basel which is published as 'The collected scientific papers of the Mathematicians and Physicists of the Bernoulli family', edited by David Speiser on behalf of the Naturforschende Gesellschaft in Basel and the Otto Spiess Foun- 
dation. 'The development of mathematics in the 17th and 18th century has provided the foundation of our scientific age. No less than 8 members of the Bernoulli family from Basel have contributed significantly to the rise of science. The Bernoulli Edition aims at rendering accessible to the modern reader their works (some of which are unpublished as yet) as well as their correspondence with most of the distinguished scholars of their time. Each text is presented in its original language (mostly Latin or French) and furnished with extensive commentaries. The introduction and notes are in the language of the respective editor (German, English, French or Italian).

Among the great editions of scientific works of the 17th and 18th centuries, the Bernoulli Edition occupies a special position; unlike, for example, the Leibniz and the Euler Edition, it is dedicated not to a single author, but to a group of nine closely related authors.

The Bernoulli Edition presents a critical, fully annotated edition of the collected works and correspondence of these authors. It includes their scientific publications, the most important of those texts which exist only in manuscript form, and an extensive selection of their correspondence, for the most part hitherto unpublished. The Bernoulli Edition establishes an authoritative version of the source texts, starting from either the original publications or the manuscripts. The commentaries facilitate access to the historical texts for the modern reader by providing interpretative introductions, explanatory notes and indexes. All texts are printed in the original language (mainly Latin and French); the commentaries are mostly in English (in the past, some volumes have been annotated in French, German or Italian). Copious illustrations present figures from original printings as well as samples from holographs. The Bernoulli Edition is divided into two sections, one for the Collected Works and one for the Correspondence. Each section is composed of several series, each of these being dedicated to one or, in some cases, to two of the nine authors.' (The leaflet reflects this arrangement.) 
The following 5 volumes of the collected works have appeared with Birkhauser Verlag, Basel, and further volumes are in preparation.

Vol. 1. Astronomie, Philosophia naturalis, edited by J O Fleckenstein, 1969 (2nd printing 1989) 541 pages, 224 ills.

Vol. 2. Elementarmathematik, edited by C S Roero and T Viola, 1969, 698 pages, 291 ills.

Vol. 3 Wahrscheinlichkeitsrechnung, edited by B L van der Waerden, 1975, 594 pages, 34 ills.

Vol. 4 Reihentheorie, edited by A Weil, 1993, 320 pages, 40 ills.

Vol. 5 Differentialgeometrie, edited by A Weil and M Mattmuller, 1999, 472 pages, 186 ills.

Vol.6 (in preparation) Mechanik, edited by P Radelet-de Grave and D Speiser.

Acknowledgement: The support from the Bernoulli Edition in Basel is greatly appreciated.

\section{Suggested Reading}

Address for Correspondence Wolfgang Polasek Wirtschaftswissenschaftliches Zentrum Statistik und Okonometrie University of Basel, Holbeinstr. 12, $\mathrm{CH}-4051$ Basel, Switzerland. Email: Wolfgang.Polasek@unibas.ch
[1] Daniel Bernoulli, De mensura sortis, Econometrica, 22, 23-36, 1954.

[2] C C Gillispie (ed.), Dictionary of Scientific Biography, Vol. 2 (H BergerCh. Buys Ballot), New York: Charles Scribner's Sons, New York, 1970.

[3] G Shaffer, The Bernoullis in Encyclopedia of Statistical Sciences, $S$ Kotz and N L Johnson (eds.)Wiley, New York, 1982.

[4] G Shaffer, The significance of Jacob Bernoulli's Ars Conjectandi for the philosophy of probability today, fournal of Econometrics, 75, 15-32, 1996.

[5] B L van der Waerden, (ed.) Die Werke von Jacob Bernoulli, Vol. 3, Wahrscheinlichkeitsrechnung, Birkhauser Verlag, Basel, 1975.

[6] S M Stigler, The history of statistics: The measurement of uncertainty before 1900, Harvard University Press, Cambridge, 1986.

[7] S M Stigler, The Bernoullis of Basel, foumal of Econometrics, 75, 7-14, 1996. 